\title{
Un univers en mutation rapide
}

L'évolution de la formation continue des enseignants en Angleterre

A rapidly changing universe. The evolution of in-service training of teachers in England

Un universo en rápido cambio. La evolucíon de la formacíon permanente de los docentes en Inglaterra

\section{Muriel Robinson}

Traducteur : Jean-Paul Gratias

\section{OpenEdition} Journals

Édition électronique

URL : http://journals.openedition.org/ries/2791

DOI : $10.4000 /$ ries. 2791

ISSN : 2261-4265

Éditeur

Centre international d'études pédagogiques

Édition imprimée

Date de publication : 1 mars 1999

Pagination : 37-52

ISBN : 9771254459005

ISSN : 1254-4590

\section{Référence électronique}

Muriel Robinson, «Un univers en mutation rapide », Revue internationale d'éducation de Sèvres [En ligne], 21 | mars 1999, mis en ligne le 28 mai 2013, consulté le 07 janvier 2020. URL : http:// journals.openedition.org/ries/2791; DOI : 10.4000/ries.2791

Ce document a été généré automatiquement le 7 janvier 2020.

(c) Tous droits réservés 


\section{Un univers en mutation rapide}

L'évolution de la formation continue des enseignants en Angleterre

A rapidly changing universe. The evolution of in-service training of teachers in England

Un universo en rápido cambio. La evolucíon de la formacíon permanente de los

docentes en Inglaterra

Muriel Robinson

Traduction : Jean-Paul Gratias

1 Le tableau que je dresse ici des dispositions actuelles et anciennes n'est pas la vision détachée et objective d'un observateur extérieur qui se serait livré à des recherches minutieuses. C'est le compte rendu d'une expérience vécue, fondé sur mes rencontres personnelles avec l'univers de la formation professionnelle continue ${ }^{1}$, tout d'abord comme institutrice d'école primaire, puis, ces douze dernières années, comme intervenante dans la formation initiale puis dans la formation continue des enseignants.

2 Comme dans toute expérience vécue, ma vision est partiale et l'expérience elle-même est chaotique et inachevée. Les vues que j'expose ne représentent en aucune façon la position officielle de mon université, mais bien les spéculations et les réflexions que m'inspirent mes efforts personnels pour rationaliser l'univers en mutation rapide dans lequel je travaille.

\section{D'où vient le système actuel ?}

Lorsque j'ai commencé à enseigner, en 1976, la formation continue dispensée aux enseignants anglais souffrait d'un manque évident d'organisation, surtout en ce qui concerne la participation des établissements d'enseignement supérieur. Mon propre institut de formation devait bien proposer des stages pour les enseignants titulaires, car je me souviens effectivement que ceux d'entre nous qui repartaient avec leur certificat en poche connaissaient l'existence d'une version à temps partiel du diplôme de Bachelor of Education que certains préparaient au cours de la quatrième année. Mais je 
ne pense pas que l'on nous ait jamais donné de détails à ce sujet, ni que l'on nous ait jamais dit qu'il existait, dans mon institut ou ailleurs, des moyens de compléter notre formation initiale.

$\mathrm{Au}$ cours des vingt dernières années, de nombreux changements se sont produits. Progressivement, le nombre d'enseignants demandeurs de formation complémentaire a augmenté, tandis que se modifiait la perception générale de la formation professionnelle, dont personne ne conteste plus, à présent, qu'elle doive se poursuivre sans interruption depuis la formation initiale jusqu'à la retraite. Cependant, des modifications récentes et résolument radicales de la politique gouvernementale ne vont pas manquer de provoquer des changements encore plus rapides, qui n'auront peut-être pas tous des effets positifs. Pour comprendre les implications de ces changements, il est nécessaire d'étudier comment façon la formation continue a été financée et réglementée jusqu'à aujourd'hui.

\section{Les priorités des centres pédagogiques}

5 Traditionnellement, le niveau le plus élémentaire de formation professionnelle continue (CPD) a toujours été dispensé par des centres pédagogiques administrés par les Local Education Authorities ${ }^{2}$ (LEA), elles-mêmes dirigées par des conseillers locaux élus. Ces centres pédagogiques proposaient toutes sortes de choses, depuis les prêts de matériel et les séances ponctuelles sur la confection de décorations de Noël jusqu'à des stages plus substantiels, souvent validés par des organismes extérieurs. Par exemple, au début des années quatre-vingt, j'ai suivi une formation à temps partiel, étalée sur toute l'année, consacrée à l'utilisation de la photographie à l'école; elle était organisée par les centres pédagogiques de trois communes voisines et validée par la Royal photographie Society. Des stages comme celui-là, financés par le budget des LEA, ne coûtaient rien aux enseignants.

Ces dernières années, les crédits alloués à ces centres ont connu un déclin constant, en partie à cause de restrictions budgétaires généralisées, mais aussi en raison des changements survenus dans les structures éducatives ${ }^{3}$. Tandis que certaines écoles, dont le statut était modifié, fonctionnaient à l'aide de subventions provenant directement du ministère, le budget des autorités locales était réduit en proportion. Dans le même temps, on demandait aux LEA de consacrer aux écoles une partie beaucoup plus importante du budget central, y compris les crédits de la formation continue, si bien qu'il leur a été de moins en moins possible de s'assurer les services des professeurs formateurs qui, traditionnellement, organisaient et assuraient les stages des centres pédagogiques, et de consacrer les crédits d'État au financement de ces stages.

7 Dans les villes où ils ont survécu, les centres pédagogiques ont dû adapter considérablement leur rôle et acquérir, pour subsister, de nouvelles compétences en matière financière. Le coût des formations est désormais calculé au plus juste et la plupart d'entre elles ont cessé d'être gratuites pour les enseignants. Les conseillers pédagogiques ont fort à faire avec les tâches d'inspection et disposent de peu de temps pour organiser des stages; les professeurs formateurs, souvent engagés pour des contrats de courte durée, doivent travailler dans les limites d'un budget serré et apporter la preuve que les stages sont rentables. Certains d'entre eux doivent assurer des rentrées d'argent pour couvrir le montant de leur salaire ${ }^{4}$. 
8 Cela ne revient pas à dire que le «bon vieux temps " était une sorte d'âge d'or de la formation, ni à suggérer qu'il n'y a rien de valable dans tous les changements mis en œuvre. Autrefois, on ne contrôlait guère la qualité des formations et on cherchait rarement à évaluer leur efficacité en termes d'amélioration des pratiques pédagogiques. On aidait rarement les enseignants à découvrir le meilleur moyen de tirer parti de leurs qualités ou d'amender leurs insuffisances et on ne prenait pas nécessairement les dispositions souhaitées, pour pourvoir aux priorités locales ou nationales. Cependant, il n'est pas possible de dénier toute valeur à cet ancien système ; ce dont nous avons besoin, c'est d'une évaluation détaillée des points forts et des faiblesses du système actuel, qui trouve manifestement ses racines dans un contexte historique précis, et ceci sans dénigrer ni encenser les pratiques passées.

\section{Le rôle des établissements d'enseignement supérieur ${ }^{5}$}

Parallèlement aux structures mises en place dans les centres pédagogiques, le secteur des universités et de l'enseignement supérieur propose également, à des niveaux variés, des formations destinées aux enseignants et, depuis toujours, un petit nombre de ces derniers en profite pour acquérir de nouvelles compétences. Certains, comme ce fut mon cas, reçurent une aide financière de leurs LEA pour suivre, à temps partiel, des cours de maîtrise. Les mieux lotis, remplacés à temps complet, purent même quitter leur salle de classe pour reprendre leurs études, changeant parfois de spécialité pour pallier une pénurie au niveau national. À la fin des années soixante-dix et au début des années quatre-vingt, par exemple, le manque de professeurs de physique a conduit l'lnner London Education authority (ILEA) à remplacer certains professeurs afin qu'ils suivent à temps complet un troisième cycle d'un an à ce qui s'appelait alors le Thames Polytechnic (et qui est devenu l'université de Greenwich). Dans l'ensemble, cependant, le choix des formations proposées devait moins à des préoccupations stratégiques qu'aux spécialités et aux traditions des institutions concernées et, bien souvent, les établissements scolaires ne mettaient guère à profit les nouvelles connaissances et compétences acquises par leurs enseignants, même lorsque c'étaient les LEA qui finançaient leur formation. Les enseignants avaient tendance à considérer leurs nouvelles qualifications comme une étape vers une promotion plutôt que comme un avantage pour leur établissement scolaire et pour leur enrichissement personnel. Certains allaient même jusqu'à garder le secret sur leurs études de peur d'essuyer les critiques et l'antagonisme de ceux de leurs collègues qui considéraient l'université et ses cours par trop éloignés des besoins et des problèmes rencontrés en classe.

Peu à peu, cette situation a évolué. De plus en plus, à mesure qu'augmente le nombre d'enseignants titulaires d'une licence (graduate), la maîtrise (master qualification) est devenue, sinon le minimum requis des futurs chefs d'établissement, du moins un titre universitaire supplémentaire souhaitable pour tous. À un niveau moindre, alors qu'autrefois il suffisait de suivre des cours à l'université, il semble qu'aujourd'hui un titre universitaire quelconque soit nécessaire pour confirmer cette formation aux yeux des conseillers pédagogiques et des commissions de validation. Dans les établissements secondaires, au moins, le niveau de la maîtrise est à présent considéré comme incontournable. De ce fait, que les enseignants complètent leur formation pour des raisons purement pratiques ou pour satisfaire un réel désir de se cultiver, ils ne sont plus considérés comme des spécimens aberrants de la profession. Dans le même temps, les établissements d'enseignement supérieur ont fourni de gros efforts pour augmenter 
leurs parts de marché dans le domaine de la formation professionnelle continue. Redéfinissant leurs priorités, ils se sont attelés au problème, bien réel dans une société où les employeurs ont de plus en plus d'exigences, des salariés qui poursuivent des études, en adoptant des horaires plus flexibles et une approche modulaire qui s'appuie sur leur expérience professionnelle et va même jusqu'à la prendre en compte. Cependant, comme dans le cas des nouvelles orientations des LEA, il ne s'agit là ni du meilleur des mondes ni d'un système financièrement exsangue et, ce dont nous avons une fois de plus besoin, c'est d'une évaluation et d'une remise en question de la politique et des pratiques actuelles.

\section{L'obsession de la rentabilité}

11 S'il est un point particulièrement positif, c'est que ces changements s'accompagnent de l'émergence d'une nouvelle série de partenariats entre les LEA et les établissements d'enseignement supérieur, des partenariats qui, en même temps, exercent une influence sur les dits changements et sont influencés par eux. Ceci résulte en partie d'une convergence d'intérêts communs: le supérieur, employant davantage d'enseignants qui ont une expérience récente de la conduite d'une classe, a réexaminé les besoins en formation continue des collègues des établissements scolaires et, pour leur part, les conseillers des centres pédagogiques locaux ont cherché à établir un cursus de formation plus complet et plus systématique des personnels. Mais il existe aussi à cette tendance une raison financière qui mérite d'être expliquée.

Une série de restrictions budgétaires ${ }^{6}$ a conduit les universités et les anciens polytechnics à prendre conscience des nouvelles réalités financières et à tenir plus clairement compte des critères de rentabilité. Dans le même temps, les remplacements des enseignants à temps plein ont, de fait, disparu. Organiser, avec un budget restreint, des cours à temps partiel pour de très petits groupes d'étudiants nécessitant un taux élevé d'heures/professeurs, implique des contraintes qu'il a bien fallu prendre en compte et il en résulte une attitude nouvelle qui reconnaît clairement l'importance d'une solide étude de marché et d'un recrutement efficace ${ }^{7}$. Les exigences des divers organismes de financement, réclamant des rapports de plus en plus détaillés (ce qui, en ce qui concerne les étudiants à temps plein, peut même entraîner une réduction des crédits alloués aux institutions en sous-effectifs), ont exacerbé cette nouvelle obsession de la rentabilité.

De la même façon, lorsque les LEA furent confrontées à la nouvelle situation financière de la gestion locale des établissements scolaires ${ }^{8}$ et, en particulier, aux conditions à remplir pour obtenir le financement complémentaire fourni par les bourses du ministère de l'Éducation pour la formation, elles découvrirent d'excellentes raisons, pédagogiques mais aussi financières, pour travailler en partenariat. De nombreuses bourses gouvernementales mettaient les LEA dans l'obligation de s'associer à un établissement d'enseignement supérieur chargé d'accréditer les formations financées de cette façon, encore que, jusqu'à une date très récente, la réalité de ces associations et leur efficacité en termes de contrôle de qualité n'aient pratiquement fait l'objet d'aucune vérification. 


\section{Et maintenant?} longue commencent, sans doute, à se demander ce qu'il y a de si important dans ces évolutions et modifications graduelles et je leur donnerais raison si rien d'autre n'avait changé. Très récemment encore, l'évolution en cours semblait concerner une conception plutôt raisonnable du partenariat, une prise de conscience accrue des réalités économiques et, par-dessus tout, une véritable volonté d'améliorer la qualité de l'enseignement, pour réagir au flot incessant des critiques d'un public dont la cible est le niveau des élèves et les membres de la profession. Les changements intervenus furent principalement le fait d'individualités très impliquées dans leur mission d'enseignant, en réaction à tout un ensemble de facteurs extérieurs ou bien que motivaient leur désir implicite de se perfectionner. Cependant, la situation a sensiblement évolué depuis environ dix-huit mois, en raison d'une série de décisions provenant du ministère (et dont les mobiles sont apparemment politiques) : ce sont ces dernières modifications que je me propose d'examiner à présent, compte tenu du contexte décrit ci-dessus.

\section{L'élaboration d'une plate-forme de référence}

Sous le gouvernement précédent - conservateur - fut créée la Teacher Training Authority (TTA), destinée à devenir un organisme central chargé d'examiner tous les aspects du recrutement et de la formation (à la fois initiale et continue) des enseignants. Le nouveau gouvernement travailliste a reconduit la TTA, étendant ses attributions ${ }^{9}$ et ses pouvoirs, loin d'être affaiblis, ont été plutôt renforcés. Les membres de la TTA (qui sont nommés et non pas élus) sont chargés de contrôler à la fois le budget de la formation initiale et celui de la formation continue qui dépendaient auparavant, en partie du moins, de l'organisme de financement de l'enseignement supérieur. Il en résulte que la TTA détient un pouvoir considérable. Il y aurait beaucoup à dire sur la façon dont fonctionne cette institution, mais cela dépasserait le cadre de notre sujet. J'aimerais simplement mettre en évidence trois aspects du rôle joué par la TTA, afin de montrer qu'ils ont eu (et continueront d'avoir) un impact considérable sur les formes prises en Angleterre par la formation continue, et d'évaluer dans quelle mesure cet impact est positif.

\section{Critères nationaux et priorités de la formation continue}

La TTA a publié les critères correspondant à différents profils d'enseignants. Les premiers critères qui concernent les enseignants nouvellement certifiés ${ }^{10}$, définissent l'objectif à atteindre au cours de la formation initiale ; ils sont consignés dans le Career Entry Profile ${ }^{11}$, un document aussi conçu pour aider les nouveaux professeurs à cerner leurs besoins en matière de formation continue. Il existe des critères pour les chefs d'établissement et un diplôme pour les enseignants qui veulent le devenir, le National Professional Qualification for Headteachers (NPQH), qui deviendra à terme une qualification obligatoire. D'autre part, il y a des critères précis pour chaque niveau de responsabilité, y compris pour les coordinateurs en éducation spécialisée et pour les responsables d'équipes pédagogiques de chaque matière, pour lesquels le ministère n'a 
pas encore défini de diplôme spécifique. Dans la plupart des cas, la liste de ces critères a d'abord circulé pour consultation, à l'état de projet, avant de recevoir sa forme définitive.

À partir de ces critères, et des problèmes qui se posent dans le système scolaire avec les structures actuelles, la TTA a déterminé pour la formation continue un ensemble de priorités, à l'échelon national, qui deviennent de plus en plus une plate-forme de référence, contrôlant quel type de formation sera ou non financé. Ces priorités prendront effet lorsque commencera à fonctionner le premier cycle de cours financés après appel d'offres (automne 1998). D'ores et déjà, cependant, l'existence du projet de priorités nationales a modifié les structures en place. Les priorités actuelles, déterminées pour les trois ans à venir (en tenant compte malgré tout d'une clause permettant à la TTA de les modifier et de les compléter), sont centrées sur les préoccupations du gouvernement actuel.

\section{Formations retenues en priorité pour l'obtention d'une bourse de la TTA}

Les formations retenues en priorité pour l'obtention d'une bourse de la TTA ont été recensées après examen des rapports de recherche et d'inspection, comprenant une enquête de la TTA sur les LEAs, les établissements d'enseignement supérieur et les associations professionnelles et spécialisées. Elles concernent :

- la direction et la gestion des établissements ;

- l'enseignement spécialisé en école primaire, axé principalement sur la maîtrise de la lecture et du calcul

- l'étude approfondie de la matière enseignée pour les enseignants du primaire, particulièrement en maitrise de la langue, mathématiques, sciences, dessin et technologie ;

- l'enseignement aux très jeunes enfants;

- l'enseignement aux élèves de 14 à 19 ans ;

- l'initiation à l'informatique ;

- la formation à l'éducation spécialisée (particulièrement pour les enseignants dont les élèves ont des besoins significatifs dans ce domaine et pour les coordinateurs en éducation spécialisée de l'enseignement général);

- la gestion du comportement des élèves ;

- l'amélioration à long terme du système éducatif ;

- les formations conformes aux critères fixés par la TTA pour les enseignants et les chefs d'établissement.

Ces priorités ne sont pas incompatibles. Elles seront réexaminées de façon continue et leur choix sera sujet à des modifications périodiques.

Extrait de : Annex B, Invitation to Bid for TTA INSET Funds, publié par la TTA en novembre 1997

La maîtrise de la lecture, de l'écriture et du calcul y figure en bonne place, de même que l'informatique, la télématique et les structures pour les 14-19 ans. Les besoins de l'éducation spécialisée arrivent en tête de liste, de même que la gestion des établissements scolaires et l'amélioration de leur fonctionnement. Bien que personne ne puisse nier l'importance de ces questions, le fait qu'elles aient été choisies dans le vaste ensemble que constitue le National Curriculum et celui, plus large encore, des besoins en matière d'éducation a créé une approche à deux niveaux qui rend difficile le traitement d'autres problèmes. L'éducation physique, les arts plastiques et la musique ont été oubliés, par exemple ; l'anglais et les mathématiques constituent des domaines plus vastes que la maîtrise de la lecture et du calcul. Il est trop tôt pour déceler une tendance nette à l'échelon national mais, dans mon université, assurément, notre programme reflète déjà l'influence de ces priorités et il continuera probablement de se modifier, à mesure que les structures mises en place par la TTA prendront une part plus grande dans notre budget. 


\section{Qui décide quoi ?}

19 La question cruciale qui se pose ici est de savoir comment ces priorités ont été définies et qui décide du contenu de la plate-forme de référence. La réponse semble être qu'il existe une volonté politique délibérée remettant en cause la tradition d'indépendance de l'enseignement supérieur. Pour de nombreuses raisons, on pourrait prétendre que l'indépendance de l'université n'est plus souhaitable ${ }^{12}$. Une plate-forme conçue par un groupe d'universitaires éloignés des réalités de la vie quotidienne ne serait pas meilleure et risquerait même d'être plus mauvaise qu'un modèle issu de mobiles politiques. Cependant, les instituts actuels de formation pédagogique n'ont rien d'une tour d'ivoire. Les récents changements de personnels - qui nécessitaient le recrutement d'un nombre important de nouveaux professeurs ayant une expérience récente et appropriée et conservant, une fois en poste, de nombreux contacts avec les établissements scolaires - ont revigoré de nombreux départements qui souffraient de la stagnation postérieure à la publication du rapport James ${ }^{13}$. Dans ma propre université, qui ne constitue en aucune façon un cas unique, les contacts avec l'enseignement primaire et l'enseignement secondaire ne manquent assurément pas. Non seulement les membres de nos équipes visitent les établissements pour rencontrer les élèves, mais ils jouent également le rôle de membres du conseil d'établissement, interviennent euxmêmes dans les classes et travaillent avec des enseignants en poste, pour sonder les professeurs recrutés de fraîche date et mettre au point de nouvelles formations. Les liens avec les LEA sont plus forts que jamais et la prise de conscience des besoins locaux en matière de formation continue est très forte et repose sur des réalités tangibles. Pourtant, les priorités ministérielles n'ont pas été établies à la suite d'une véritable concertation avec ceux d'entre nous qui sont partie prenante dans cette question, mais après une consultation purement symbolique et dans un état d'esprit général qui évoque un lien étroit avec la politique et la rhétorique du parti travailliste. Tant que ces priorités n'auront pas été réévaluées en commun par tous les acteurs concernés, on se posera la question de savoir pour qui elles constituent des priorités, pourquoi elles ont été choisies de préférence à d'autres et dans quelle mesure, elles permettront un véritable progrès de l'enseignement en Angleterre ${ }^{14}$.

\section{Financement et clientèle des formations}

Dans le sillage de l'élaboration de la plate-forme de référence et afin de faire en sorte que cette plate-forme soit prise au sérieux par les organismes de formation continue, nous avons assisté à une modification profonde des structures de financement de ces organismes, qu'ils dépendent de l'enseignement supérieur ou des LEA. Tous les crédits gouvernementaux destinés à la formation des enseignants parviennent aux universités par le biais de la TTA plutôt que par celui de l'organisme de financement de l'enseignement supérieur. En ce qui concerne la formation professionnelle continue, ce changement est particulièrement significatif car, entre la période actuelle et l'an 2000, nous allons passer d'un système d'allocation globale de bourses d'études (que l'établissement bénéficiaire répartit selon ses propres critères de choix) à un système d'appel d'offres qui met en concurrence les divers organismes priés de proposer des formations conformes aux priorités définies par la TTA et aux objectifs nationaux. 
21 Par conséquent, pour continuer à bénéficier des crédits gouvernementaux, les universités ont dû préparer, dès cette année, des plans de formation continue pour les trois ans à venir et les résultats de l'appel d'offres leur ont été notifiés au printemps dernier. Un nombre important d'établissements qui, traditionnellement, assuraient la formation continue des enseignants, se sont vus complètement écartés. Dans le sudouest de l'Angleterre, par exemple, sur une dizaine d'institutions concernées, seule ma propre université a vu son projet accepté. Le tollé déclenché par la publication des résultats a permis d'obtenir un second appel d'offres, plus limité, destiné à combler quelques lacunes géographiques, mais il n'en reste pas moins que dans certaines universités, il va falloir prendre des décisions difficiles concernant la formation continue dès lors que les frais de scolarité des étudiants ne seront plus pris partiellement en charge par une bourse gouvernementale.

Bien que les modifications budgétaires doivent, en principe, être programmées sur trois ans, la situation s'est révélée fort différente dans la pratique. Cette année, celle de l'entrée en vigueur du nouveau système, les engagements pris étaient censés assurer un financement égal à $75 \%$ de l'allocation précédente, le choix des bénéficiaires étant laissé à la discrétion des organismes de formation. Cependant, le budget global a été réduit à la suite d'un nouveau calcul redéfinissant quelles personnes sont susceptibles de recevoir de la TTA une bourse de formation - les bourses de la TTA ne peuvent être attribuées qu'aux enseignants possédant le statut de professeur certifié (QTS) ${ }^{15}$ - et lesquels peuvent en recevoir une de l'organisme de financement du supérieur qui détient et distribue le reste des fonds disponibles. Le déficit résultant de cette réduction a été comblé, pour chaque établissement, par une augmentation du budget alloué par l' Higher Education Funding Council, mais cette somme ne sera pas forcément réinjectée dans la formation des maîtres ${ }^{16}$. Dans presque tous les cas, les établissements d'enseignement supérieur dont les propositions ont été retenues à la suite de l'appel d'offres recevront, malgré tout, moins d'argent pour cette année que pour la précédente et il faut ajouter à cette situation la menace d'une réduction supplémentaire de crédits, s'ils ne parviennent pas à recruter suffisamment de candidats pour leurs formations à temps partiel.

\section{Financer l'excellence}

Dans certains cas, cette restriction budgétaire (encore plus sévère, bien sûr, pour les établissements dont les offres ont été refusées) est exacerbée par une seconde modification du financement, introduite en parallèle. Le budget des LEA destiné aux bourses de formation a changé, lui aussi, de façon sensible, recevant même un nouveau nom - Standard Funding ${ }^{17}$ - qui montre une volonté nouvelle d'atteindre les critères nationaux de référence et d'évaluation, et un ensemble de priorités définies avec une plus grande rigueur. Alors que, l'an passé, mon université accréditait des stages couvrant une gamme étendue de matières enseignées, les LEA ont dû, cette année, en supprimer un nombre important, même dans des matières prioritaires telles que l'anglais, et se concentrer plutôt sur des stages beaucoup plus brefs liés à des initiatives à très court terme telles que l'«heure de lecture ». Cela s'est traduit, pour les enseignants, par la disparition de quelques possibilités de formation tout à fait valables. Par exemple, pendant quatre ans, nous avons travaillé conjointement avec la LEA du West Sussex pour proposer un programme de cent vingt heures, financé par les bourses pour la formation et destiné aux coordinateurs de l'enseignement de l'anglais en 
primaire au niveau 1 (enfants de 5 à 7 ans) et au niveau 2 (enfants de 7 à 11 ans). Ceci donnait aux enseignants l'occasion de bénéficier d'une décharge de cours pour prendre connaissance des dernières recherches en matière de pédagogie, étudier de nouveaux supports et discuter entre eux des nouvelles avancées. Ils recevaient une formation dispensée conjointement par les conseillers pédagogiques locaux et les enseignants de l'université, constatant par là même une véritable collaboration entre les LEA et le supérieur. Ceux d'entre nous qui intervenaient dans ces formations y trouvaient un bénéfice supplémentaire avec la possibilité de nouer de nouveaux liens avec l'école et d'être plus au fait des pratiques pédagogiques que l'on y rencontre. Les nouvelles dispositions budgétaires interdisent aux LEA de continuer à financer un stage qui se révélait relativement coûteux, brisant du même coup ces liens extrêmement précieux tout en réduisant un peu plus encore nos revenus.

\section{Des clientèles exclues}

Ces changements structurels du système de financement ont également soulevé des interrogations concernant la nature du public visé par ces stages. Ces dernières années, la plupart des institutions du supérieur proposant des formations ont adopté une définition plutôt large de la clientèle à laquelle s'adressait la formation continue en pédagogie - tendance encouragée par les incitations ministérielles à faciliter l'accès aux formations universitaires, particulièrement pour les catégories sociales traditionnellement sous-représentées. Le fait qu'il y ait, d'un côté, une volonté sincère d'ouverture de l'université et, de l'autre, un besoin réel de recruter de nouveaux effectifs a conduit à une modification de la clientèle qui correspond mal aux critères retenus par la TTA pour accorder une bourse de formation.

Dans ma propre université, nous définissons le cadre de la formation continue en matière de pédagogie comme s'adressant à toute personne impliquée dans l'enseignement ou l'apprentissage sur le plan professionnel ou personnel. Nous travaillons, bien évidemment, avec de nombreux enseignants certifiés (QTS) en exercice, mais aussi avec toutes sortes de groupes socioprofessionnels et d'individualités isolées. Conférenciers de l'éducation continue, formateurs des écoles d'infirmières et personnel non-enseignant (puéricultrices, techniciens, assistants) du secteur public représentent un pourcentage assez élevé de notre clientèle, de même que les enseignants, certifiés (QTS) ou non, du secteur privé, les responsables de la formation des personnels d'entreprise et les personnes qui se consacrent à l'enseignement bénévole dans le cadre de leurs églises. Nous avons également vu passer dans nos stages des membres de conseils d'administration d'établissements scolaires, des moniteurs d'auto-école, des officiers de police chargés de formation et des parents d'élèves et, dans nos propres rangs, beaucoup d'entre nous ont suivi ce même parcours pour obtenir une maîtrise (master qualification). Les bourses de la TTA ne peuvent servir à financer, par conséquent, qu'une partie de nos formations traditionnelles. Il est possible de rassembler dans un même groupe des stagiaires boursiers et d'autres qui ne le sont pas, mais les non-boursiers doivent soit avoir recours à une aide prélevée sur le restant du budget de l'université, soit prendre à leur charge le coût total du stage.

Il est, bien sûr, tout à fait compréhensible que la TTA, qui est un organisme d'État, refuse d'apporter une aide financière à certains des groupes cités ci-dessus qui ne jouent aucun rôle dans l'enseignement public. Cependant, dans le cas des personnes qui 
travaillent dans le secteur public sans avoir le Qualified Teacher Status (les puéricultrices, par exemple), cette décision contredit la logique de partenariat entre diverses professions, officiellement encouragée. La difficulté de distinguer les différents stagiaires au moment de leur évaluation et à identifier les différentes sources de financement pourrait bien conduire à une réduction du nombre des formations proposées ou, au mieux, à une tendance qui consisterait à organiser des formations séparées plutôt que de rassembler des groupes différents pour confronter leurs points de vue. Si nous considérons l'une des priorités gouvernementales - l'amélioration de la discipline dans les établissements -, l'artificialité et la faiblesse d'une telle coupure saute aux yeux. Par exemple, l'un des éléments clés du bon fonctionnement d'un code de discipline, peu de gens le contesteront, est la cohérence de la démarche. Quel meilleur moyen de garantir cette cohérence que d'offrir un stage qui regroupera toutes les acteurs concernés dans l'implantation du "code de discipline», depuis les surveillants de cantine et les assistants jusqu'à l'encadrement administratif, afin qu'ils découvrent ensemble les dernières recherches en la matière, qu'ils en débattent et qu'ils exposent des approches plus efficaces? Avec le nouveau système, qui a pourtant fait de cet aspect de la vie scolaire une de ses priorités, ce genre de rencontre a beaucoup moins de chances de se produire qu'auparavant.

\section{Centralisation et incohérence}

27 En plus de l'exclusion d'une certaine partie de la clientèle, les nouvelles dispositions ont fait des coupes sombres dans les structures régionales de formation. Sur les soixante-neuf institutions existantes qui ont répondu à l'appel d'offres de la TTA, trente-deux seulement ont vu leur projet retenu. Ce choix a été fait sans prendre en considération leur implantation géographique et de nombreux secteurs vont constater une diminution brutale du nombre de structures, au cours des prochaines années. La seconde vague d'appel d'offres comblera certaines des lacunes les plus criantes, mais de nombreux enseignants se verront contraints de parcourir des distances plus importantes pour participer à des stages de formation continue en université. Compte tenu du blocage des crédits d'équipement évoqué plus haut et du fait que les bourses de la TTA ne couvrent qu'une partie des frais d'inscription aux formations - ce qui contraint les enseignants à payer jusqu'à 2600 livres de leur poche pour des études de maîtrise (master degree) - les conditions actuelles ne les motivent guère à entreprendre une formation de longue durée. Ceci est d'autant plus vrai dans l'atmosphère de découragement général que connaissent les enseignants anglais, qui viennent de subir dix ans de changements rapides, une bureaucratie de plus en plus pesante, les critiques de l'opinion publique et la baisse de leurs traitements. L'objectif, fort louable, d'utiliser les réformes pour rendre la formation des professeurs plus efficace et pour améliorer la qualité de l'enseignement qu'ils dispensent risque ainsi d'être sabordé par un système d'appel d'offres mal conçu et un budget sous-dimensionné.

Certes, dans la situation antérieure aux appels d'offres, l'implantation régionale des structures de formation n'était pas, non plus, organisée ni planifiée en tant que telle et une réduction progressive du nombre d'établissements d'enseignement supérieur procurant une formation pédagogique initiale a fini par appauvrir la répartition géographique des centres de formation. Mais la situation présente a ceci d'ironique que l'introduction d'un système qui est, par bien des aspects, plus centralisé et plus rationnel a conduit, en fait, à une structure beaucoup plus disparate. 


\section{L'OFSTED $^{18}$ et l'exigence de rendement}

Le troisième facteur significatif qu'il me semble important de prendre en considération ici est la rigueur croissante du processus d'inspection dans tous les secteurs de l'enseignement. Les cinq dernières années ont vu un accroissement rapide à la fois du nombre d'inspections des structures de formation initiale des enseignants et de la sévérité des dites inspections mais, jusqu'à maintenant, la formation professionnelle continue a été très peu affectée par ce phénomène. Une conséquence directe du nouveau processus d'appel d'offres est que les organismes retenus vont être inspectés au cours des deux années à venir bien que, jusqu'à présent, les critères d'inspection n'aient toujours pas été communiqués.

Le nouveau processus et l'implication de l'OFSTED impose donc aux organismes de formation une exigence de rendement. Étant donné que ces structures sont financées par des fonds publics, il est légitime que des vérifications soient effectuées pour garantir que cet argent est dépensé à bon escient. Il est bon, également, que l'on prenne de plus en plus conscience de la nécessité d'un suivi après une période de formation pour voir de quelle façon les pratiques de classes en ont bénéficié. Cependant, le processus n'est pas simple. Tout d'abord, l'exigence de rendement constitue une nouvelle atteinte à la tradition d'indépendance des universités. Alors que, dans le passé, les universités pouvaient se permettre de rester à l'écart du courant et de critiquer les choix de politique éducative, si l'organisme qui définit cette politique devient également celui qui inspecte les structures de formation continue, le danger est grand que les universités ne puissent plus dispenser des cours qui soient de véritables remises en question, mais doivent se contenter de refléter la ligne du parti.

3 De plus, apporter la preuve que la formation continue est bénéfique n'est pas chose facile, car de nombreux autres facteurs entrent en ligne de compte ${ }^{19}$. Un enseignant peut fort bien désirer instaurer des changements dans sa classe et pourtant découvrir que les moyens nécessaires ne sont pas disponibles, que l'administration de l'établissement refuse de le soutenir ou que ses collègues sapent ses efforts. Tel autre à qui ne manqueront ni les moyens ni l'aide de ses collègues constatera peut-être qu'il est difficile, soit de déceler des améliorations, soit de déterminer si elles sont dues à ses innovations ou à d'autres facteurs. Dans un troisième cas de figure, on pourra constater des preuves tangibles d'un réel progrès et, pourtant, il sera encore plus difficile de déterminer précisément la relation de cause à effet - post hoc, propter hoc est une hypothèse particulièrement risquée ici. La formation d'un enseignant n'est qu'une des multiples influences qui agissent de concert pour favoriser ou freiner son évolution et il n'existe pas de réponses simples à la question épineuse de l'évaluation de l'efficacité d'une formation pédagogique.

Pour l'instant, il faut noter que les premiers échos du processus d'inspection laissent entrevoir une prudence de circonstance de la part de la TTA et de l'OFSTED. On a demandé aux universités de recenser les éléments de leur travail dont ils se sentent suffisamment sûrs pour accepter d'être jugés à travers eux et cette requête s'étend au domaine de la formation pédagogique dont elles doivent préciser quels aspects présentent des critères d'excellence. Cependant, nous n'en sommes qu'aux premiers jours du processus et aucune grille de référence n'a encore été publiée - l'histoire continue. À mesure que nous en tournerons les prochaines pages, nous saurons si 
l'intervention d'un organisme extérieur a quelque chose à nous apprendre, mais que la réponse nous plaise ou non, il est certain que seront prononcés des jugements qui influenceront en bien ou en mal les futures allocations de crédits.

\section{"Watch this space, as they say »}

33 Ces profonds changements des structures de la formation professionnelle continue en Angleterre commencent à peine à produire leurs effets et les spéculations que je viens d'exposer ne peuvent être autre chose, par conséquent, que des spéculations. Il est trop tôt pour savoir si ces évolutions resteront dans l'histoire comme le grand tournant d'une amélioration substantielle de l'enseignement public ou comme un détour nous éloignant des véritables problèmes du moment. J'espère, cependant, que cet article aura au moins montré qu'il est nécessaire, pour les universités, de procéder à un examen critique de la politique de formation des enseignants et de défendre le travail qu'elles ont accompli dans le passé, lorsque celui-ci, à son tour, a survécu au même examen critique. Comme on dit à la radio, restez à l'écoute...

\section{NOTES}

1. Continuous professional development.

2. Instances éducatives locales.

3. Pour un compte rendu exhaustif des modifications des structures et du financement des instances éducatives locales, voir Harold Heller \& Peter Edwards, Policy and Power in Education : the Rise and Fall of the LEA, London, Routledge, 1992, particulièrement le chapitre V.

4. Harold Heller \& Peter Edwards décrivent également très bien le rôle actuel des conseillers pédagogiques, p. 219-220, en ne laissant aucun doute sur la multiplicité de leurs fonctions.

5. Higher Education Institutions (HIEs).

6. Les changements de structure et les restrictions budgétaires des années quatre-vingt sont détaillés dans le rapport Higher Education in the Post-Binary System, London, Gresham Collège, Gresham Conférences on Higher Education, 1993.

7. Le système de financement autrefois en place dans les établissements supérieurs est clairement expliqué dans le chapitre 4, "The Costing and Financing of Part-Time Degree Provision ", de David Smith \& Michael Saunders, Other Routes : Part-time Higher Education Policy, London, SRHE/Open University Press, 1991.

8. Local Management of School (LMS).

9. «La mission de la Teacher Training Authority est de faire progresser le niveau des élèves, en améliorant la qualité de la formation, de l'enseignement, de la gestion des établissements scolaires, et en revalorisant le statut du corps enseignant et son image. », Anthea Millett (Chief Executive), Teacher Training Agency Annual Review, 1997.

10. Les enseignants ayant obtenu le Qualified Teacher Status (QTS).

11. Profil de début de carrière.

12. On trouvera une analyse utile de la conception traditionnelle de l'indépendance de l'université dans William Melody, «Universities and Public Policies » in Anthony Smith \& Frank 
Webster (eds), The Postmodern University? Contested Visions of Higher Education in Society, London, SRHE/Open University Press, 1997.

13. Au début des années soixante-dix, le rapport James s'est penché sur l'avenir de la formation des enseignants ; ses conclusions et les réformes qui finirent par retirer la responsabilité de cette formation aux LEA pour la confier à l'enseignement supérieur est abondamment argumentée dans Paul Sharp, The Creation of the Local Authority Sector of Higher Education, London, Falmer Press, 1987.

14. Il est intéressant de noter que le rapport du National Committee of Inquiry into Higher Education, commandité par le gouvernement, milite pour le maintien de l'indépendance de l'université vis-à-vis de toute intervention directe du pouvoir central: "Nous accordons une grande importance au maintien de cette pratique établie de longue date qui consiste à tenir le gouvernement à distance respectable des établissements supérieurs et par conséquent à conserver des organismes intermédiaires de financement», $\$ 120$. Comme la TTA n'est pas constituée de politiques élus, le poids du ministère sur le choix des membres semblerait compromettre ce principe.

15. «Le statut de professeur certifié (Qualified Teacher) s'obtient après suivi avec succès d'une formation pédagogique dans une institution accréditée d'Angleterre ou du Pays de Galles, soit concurremment, soit ultérieurement à l'obtention d'une licence dans une université britannique ou tout établissement d'enseignement supérieur habilité à décerner des diplômes. » National Standards for Qualified Teacher Status, London, TTA, 1998.

16. Cette augmentation, bien sûr, ne représente pas de nouvelles sommes d'argent mais une redistribution entre les chapitres budgétaires. Étant donné la réduction globale du budget du supérieur ces dernières années, qui pourrait, si les projets actuels sont mis en œuvre, représenter une division par deux du dit budget sur une période de vingt-cinq ans, cette redistribution a peu de chances de bénéficier de façon significative aux centres de formation, (voir le rapport du National Committee of Inquiry into Higher Education, p. 3)

17. Ce terme recouvre le financement des opérations permettant aux professeurs de faire atteindre à leurs élèves les objectifs du National Curriculum

18. L'Office for Standards in Education (OFSTED) est l'organisme ministériel responsable des inspections des établissements scolaires, universitaires, et des formations des enseignants. Il remplace le Corps d'inspection de Sa Majesté (Her Majesty's Inspectorate).

19. La nature complexe, problématique, des indicateurs de performance dans l'enseignement supérieur est clairement exposée dans le rapport d'une commission d'enquête sur le sujet, publié par le Polytechnics and Collèges Funding Council (qui a précédé l'organisme pour la formation initiale et continue des enseignants), Alfred Morris, Performance Indicators : Report of a Committee of Enquiry, London, 1990.

\section{RÉSUMÉS}

En Angleterre, la formation continue des enseignants est entrée dans une phase de mutation rapide. Les nouvelles structures gouvernementales en ont modifié le financement, renforçant l'obligation de résultats et le contrôle des autorités de tutelle. Le présent article rend compte de ces changements et tente d'évaluer dans quelle mesure l'objectif, l'amélioration du fonctionnement des établissements scolaires, sera atteint. 
In England, in-service teacher training has entered a phase of rapid change. The new governmental structures have changed the financing, reinforcing both the obligation to produce results and the control of the authorities in charge. This article gives an account of these changes and attempts to assess to what extent the aim of improving the functioning of schools is attained.

En Inglaterra, la formación permanente de los docentes ha entrado en una fase de cambio rápido. Las nuevas estructuras gubernamentales modificaron su financiacián, intensificando la obligación de resultados y el control de las autoridades tutelares. Este articulo da cuenta de dichos cambios y trata de calcular en que medida se alcanzará la meta: la mejora del funcionamiento de los centras docentes.

INDEX

Index géographique : Angleterre

Mots-clés : formation continue, formation des enseignants, enseignant, financement

\section{AUTEURS}

MURIEL ROBINSON

Deputy Head, School of Education, University of Brighton, Grande-Bretagne 